\title{
The Plancherel Measure for Symmetric Graphs $(*)$.
}

\author{
JaCques Faraut - Massimo A. Picardello (Roma)
}

Summary. - Let $G$ be the free product of $r$ copies of the cyclic group $\boldsymbol{Z}_{k}$. We obtain the Plancherel formula for the commutative $O^{*}$-algebra of radial convolution operators on $l^{2}(G)$. The Plancherel measure is expressed in terms of the e-function appearing in the expansion of spherioal functions on $G$ as linear combinations of exponentials.

\section{1. - Introduction.}

A function on the free group $\boldsymbol{F}_{r}$ with $r$ generators is called radial if it depends only on the length of the words. The $C^{*}$-algebra of radial convolution operators on $l^{2}\left(\boldsymbol{F}_{r}\right)$ is commutative, and its spectrum $\sigma$ supports a measure $m$ such that the $l^{2}$-norm of a radial function $f$ is the norm of its Gelfand transform $\hat{f}$ in $L^{2}(\sigma, m)$. This measure, called the Plancherel measure, has been computed in $[2,10,3,9,5]$.

The group $\boldsymbol{F}_{r}$ acts isometrically and simply transitively on the homogeneous tree $T_{d}$ with $d=2 r$. Every group acting in this way on $T_{d}$ is isomorphic to the free product $(\underset{j=1}{*} \boldsymbol{Z}) *\left(\underset{j=1}{\stackrel{*}{*}} \boldsymbol{Z}_{2}\right)$, with $2 p+s=d$. The Plancherel theorem has been extended to these groups in [1]. The Plancherel measure can be expressed in terms of the asymptotic behaviour of spherical functions on trees. Following [5], we shall denote the spherical functions by $\phi_{z}, z \in C$ : then the support $\sigma$ of the Plancherel measure is the segment $\left\{\operatorname{Re} z=\frac{1}{2}, 0 \leqslant \operatorname{Im} z \leqslant \pi / \ln q\right\}$ where $q=d-1$, and $d$ is the homogeneity degree. There exists a meromorphic function $e$ (depending on the degree), such that, except at its poles, $\varphi_{z}=c(z) h_{z}+e(1-z) h_{1-z}$ where $h_{z}(x)=q^{-z|x|}$ (here $|x|$ denotes the length of the vertex $x$ ). Then it turns out that $d m\left(\frac{1}{2}+i t\right)$ is a multiple of $\left|e\left(\frac{1}{2}+i t\right)\right|^{-2}[5,1]$. Similar relations had long been known for semisimple Lie groups.

The theory of spherical functions has been recently extended to the free products $\underset{j=1}{*} \boldsymbol{Z}_{k}(r, k, \geqslant, 2)$, which act on "symmetric graphs " [7], and on semihomogeneoos trees.

A Plancherel formula for these groups has been proved in [8], with no reference to the $c$-function. In this note we give a short and simple computation of the Plancherel measure, for groups acting on trees or on symmetric graphs (semihomogeneous

(*) Entrata in Redazione il 9 giugno 1983. 
trees), as described above, by a method which sheds light upon the role played by the $e$-function. For rank-one semisimple Lie groups, a similar approach is in [6].

For uniformity of notations, we shall always consider groups of the type $G={\underset{j=1}{r}}_{j=1}^{r} \boldsymbol{Z}_{k}$. In the case $k=2$, when the associated graph is a homogeneous tree, only trivial modifieations are needed to adapt our results to free groups and to every other group acting on the tree.

Unexplained terminology and notations are as in [7]. We were informed by W. Betori and M. PAGLiacCi of an alternative deduction of the Plancherel formula for free groups, which makes use of Radon transforms.

An extension of the results of [8] to more abstract settings was communicated to us by J. M. Cohen and A. Trenholme.

This paper has been written while the authors were visiting the Department of Mathematics of Washington University, whose warm hospitality is acknowledged with pleasure.

\section{2. - The Plancherel measure.}

Let $G=\underset{j=1}{*} \boldsymbol{Z}_{k}$, and denote by $\mu_{1}$ the probability measure equidistributed on words of length one. We shall consider the self-adjoint operator $T$, on the Hilbert space $l_{\#}^{2}$ of square-integrable radial functions on $G$, defined by $T f=\mu_{1} * f$. It has been shown in $[7$, thm. 3] that the spectrum of $T$ is the set $E \cup D$, where $E$ is the segment

$$
\left\{\lambda \in \boldsymbol{R}: \lambda=\gamma\left(\frac{1}{2}+i t\right), 0 \leqslant t \leqslant \pi / \log q\right\},
$$

and

$$
D=\left\{\lambda_{0}=\frac{1}{1-k}\right\} \text { for } k>r, D=\emptyset \text { for } k \leqslant r .
$$

(Here, as in $[7]$,

$$
\left.q=(k-1)(r-1) \quad \text { and } \quad \gamma(z)=\frac{1}{(k-1) r}\left(q^{z}+q^{1-z}+k-2\right)\right) .
$$

Let $z_{0}=(\log (k-1)+i \pi) / \log q$, so that $\gamma\left(z_{0}\right)=\lambda_{0}$. The Plancherel measure $m$ is the positive measure on the spectrum of $I$ such that, for $f \in l_{\#}^{1}$,

$$
f(e)=\int \hat{f} d m
$$

where $f(z)=\sum_{x \in G} f(x) \varphi_{z}(x)$ and $\phi_{z}$ is the spherical function. 
ThEOREM. - For every $f \in l_{\text {\#̈ }}^{1}$,

$$
f(e)=\frac{1}{2 \pi} \frac{q \log q}{(k-1) r} \int_{0}^{\pi / \log q} \hat{f}\left(\frac{1}{2}+i t\right)\left|e\left(\frac{1}{2}+i t\right)\right|^{-2} d t+\frac{q \log q}{(k-1) r} \operatorname{Res}_{z_{0}}\left[\frac{1}{c(z) c(1-z)}\right] \hat{f}\left(z_{0}\right) .
$$

(The second term on the right hand side is omitted if $k \leqslant r$.)

Proof. - Recall the following explicit expression for the $\theta$-function [7, thm. 2]:

$$
c(z)=\frac{q^{1-z}-(k-1) q^{z-1}+k-2}{(k-1) r\left(q^{-z}-q^{z-1}\right)}
$$

The resolvant $R_{\lambda}=(\lambda I-T)^{-1}$ of the operator $T$, computed in [7, thm. 3], can be expressed in terms of the $\theta$-function: For $\lambda=\gamma(z)$, Re $z>\frac{1}{2}, f \in l_{\| t}^{2}$,

$$
R_{\lambda} f=r_{\lambda} * f
$$

with

$$
r_{\lambda}(x)=\frac{1}{e(1-z)} \frac{1}{q^{z-1}-q^{-z}} q^{-z|x|}
$$

The resolvant is analytic with respect to $\lambda$ in the complement of the spectrum of $T$, and has a simple pole at $\lambda_{0}=(1-k)^{-1}$ for $k>r$. Carleman's formula $[4$, p. 920] yields a relation between $R_{\hat{\lambda}}$ and the spectral resolution $E(d \lambda)$ of $T$ : for any $f \in l_{\| t}^{2}$ and any continuous function $\varphi$ on $\boldsymbol{R}$ with compact support,

$$
\int_{-\infty}^{\infty} \varphi(\lambda)(E(d \lambda) f, f)=-p \lim _{\varepsilon \rightarrow 0^{+}} \int_{-\infty}^{\infty} \varphi(\lambda)\left(\frac{1}{2 i}\left[R_{\lambda+i \varepsilon}-R_{\lambda-i \varepsilon}\right] f, f\right) d \lambda .
$$

The measure $(E(d \lambda) f, f)$ is absolutely continuous with respect to Lebesgue meas. ure on $E$, and has an atom at $\lambda_{0}=1 /(1-k)$ for $k>r$.

We now compute its continuous part. For $\lambda=\gamma\left(\frac{1}{2}+i t\right), 0<t<\pi / \log q$,

$$
\begin{aligned}
\frac{1}{2 i}\left[r_{\lambda+i 0}(x)-r_{\lambda-i 0}(x)\right] d \lambda & =\frac{1}{2 i}\left[\frac{1}{c\left(\frac{1}{2}-i t\right)} \frac{q^{-\left(\frac{1}{2}+i t\right)|x|}}{q^{-\frac{1}{2}+i t}-q^{-\frac{1}{2}-i t}}-\frac{1}{e\left(\frac{1}{2}+i t\right)} \frac{q^{-\left(\frac{1}{2}-i t\right)|x|}}{q^{-\frac{1}{2}-i t}-q^{-\frac{1}{2}+i t}}\right] . \\
& \frac{\log q}{(k-1) r} \mid q^{\frac{1}{2}+i t}-q^{\frac{1}{2}-i t \mid} d t= \\
= & -\frac{1}{2} \frac{q \log q}{(k-1) r}\left[\frac{1}{c\left(\frac{1}{2}-i t\right)} q^{-\left(\frac{1}{2}+i t\right)|x|}+\frac{1}{c\left(\frac{1}{2}+i t\right)} q^{-\left(\frac{1}{2}-i t\right)|x|}\right] d t= \\
= & -\frac{1}{2} \frac{q \log q}{(k-1) r} \frac{1}{\left|c\left(\frac{1}{2}+i t\right)\right|^{2}} \varphi_{\frac{1}{2}+i t}(x) d t .
\end{aligned}
$$


Observing that $\left(\varphi_{z} * f, f\right)=|\hat{f}(z)|^{2}$ for $z=\frac{1}{2}+i t$, we have:

$$
\frac{1}{2 i}\left(\left[R_{\lambda+i 0}-R_{\lambda-i 0}\right] f, f\right) d \lambda=-\frac{1}{2} \frac{q \log q}{(k-1) r}\left|\bar{f}\left(\frac{1}{2}+i t\right)\right|^{2} \frac{d t}{\left|c\left(\frac{1}{2}+i t\right)\right|^{2}} .
$$

Let us assume $k>r$ and consider the singular part of $m$. We have $e\left(1-z_{0}\right)=0$, $c\left(z_{0}\right)=1 ;$ hence

$$
P_{z_{0}}(x)=q^{-z_{0}|x|}=(1-k)^{-|x|}
$$

The orthogonal projector onto the eigenspace corresponding to the eigenvalue $\lambda_{0}=\gamma\left(z_{0}\right)=\left(1-k_{i}\right)^{-1}$ is given by the residue of the resolvant $R_{\lambda}$ at $\lambda_{0}$ :

$$
\left(E\left(\left\{\lambda_{0}\right\}\right) f, f\right)=\operatorname{Res}_{\lambda_{0}}\left[\left(R_{\lambda} f, f\right)\right] .
$$

We have

$$
\operatorname{Res}_{\lambda_{0}}\left[r_{\lambda}\right]=\gamma^{\prime}\left(z_{0}\right) \operatorname{Res}_{z_{0}}\left[\frac{1}{c(1-z)} \frac{1}{q^{z-1}-q^{-z}} q^{-z|x|}\right]=\frac{q \log q}{(k-1) r} \operatorname{Res}_{z_{0}}\left[\frac{1}{c(1-z)}\right] q^{-z_{0}|x|}
$$

Therefore

$$
\left(E\left(\left\{\lambda_{0}\right\}\right) f, f\right)=\frac{q \log q}{(k-1) r} \operatorname{Res}_{z_{0}}\left[\frac{1}{c(1-z)}\right]\left|\hat{f}\left(z_{0}\right)\right|^{2} .
$$

Since $\int_{-\infty}^{\infty}(E(\lambda \hat{\lambda}) f, f)=\|f\|_{2}^{2}$, we obtain the Plancherel formula

$$
\begin{aligned}
\sum_{x \in G}|f(x)|^{2}=\frac{1}{2 \pi} \frac{q \log q}{(k-1) r} \int_{0}^{\pi / \log q}\left|\hat{f}\left(\frac{1}{2}+i t\right)\right|^{2}\left|c\left(\frac{1}{2}+i t\right)\right|^{-2} d t+ & \\
& +\frac{q \log q}{(k-1) r} \operatorname{Res}_{z_{0}}\left[\frac{1}{c(z) c(1-z)}\right]\left|\hat{f}\left(z_{0}\right)\right|^{2},
\end{aligned}
$$

where the second term has to be omitted for $k \leqslant r$.

R_малк. - The residue which yields the singular part of $m$ can be easily computed by means of (1). One obtains

$$
\frac{q \log q}{(k-1) r} \operatorname{Res}_{z_{0}}\left[\frac{1}{c(z) c(1-z)}\right]=\frac{k-r}{k} .
$$

One can also check that the expression of the Plancherel measure given in the Theorem agrees with the formulas of $[8$, thm. 2] (in our notations, the parameter $x$ which appears in these formulas becomes

$$
\left.x=q^{z}+q^{1-z}+k-2=(k-1) r \gamma(z)\right) .
$$




\section{REFERENCES}

[1] W. Betori - M. Pagliaccr, Harmonic analysis for groups acting on trees, to appear in Boll. Un. Mat. Ital.

[2] P. Cartier, Harmonic analysis on trees, Proc. Symp. Pure Math. Amer. Math. Soc., 86 (1973), pp. 419-424.

[3] J. M. CoHeN - L. DE Mrchene, The radial Fourier-Stieltjes algebra of free groups, Contemp. Math., 10 (1982), pp. 33-40.

[4] N. Dunford - J. T. SChwartz, Linear operators, Part II, Interseience, 1963.

[5] A. Figí-Talamanca - M. A. Picardello, spherical functions and harmonic analysis on free groups, J. Functional Anal., 47 (1982), pp. 281-304.

[6] M. Flensted-Jensen, Paley-Wiener type theorems for a differential operator connected with symmetric spaces, Ark. Mat., 10 (1972), pp. 143-162.

[7] A. Iozzi - M. A. Picardello, Spherical functions on symmetrie graphs, Lecture Notes in Math., 992, pp. 344-386, Springer Verlag, Berlin, 1983.

[8] G. KüHN - P. M. SOARDI, The Plancherel measure for polygonal graphs, Ann. Mat. Pura Appl., 134 (1983), pp. 393-40!̣.

[9] T. PYTLIK, Radial functions on free groups and a decomposition of the regular representation into irreducible components, J. Reine Angew. Math., 396 (1981), pp. 124-135.

[10] S. SAWYER, Isotropio random walks in a tree, Zeitsch. Wahrsch., 48 (1978), pp. 279-292. 